\title{
Avaliação dos acidentes com motocicletas no Brasil
}

\author{
Evaluation of motorcycle accidents in Brazil \\ Evaluación de los accidentes con motocicletas en Brasil
}

Recebido: 11/01/2022 | Revisado: 15/01/2022 | Aceito: 21/01/2022 | Publicado: 23/01/2022

\author{
Taís Soares Ramos \\ ORCID: https://orcid.org/0000-0002-4378-6993 \\ Universidade de Pernambuco, Brasil \\ E-mail: soaresramos9804@gmail.com \\ Katarina Haluli Janô da Veiga Pessôa \\ ORCID: https://orcid.org/0000-0002-0722-2568 \\ Universidade de Pernambuco, Brasil \\ E-mail: katarinahaluli@gmail.com \\ Ana Paula Martins de Oliveira Nascimento \\ ORCID: https://orcid.org/0000-0002-8113-7767 \\ Universidade de Pernambuco, Brasil \\ E-mail: anapaulamartins819@gmail.com \\ Caio César Gonçalves Silva \\ ORCID: https://orcid.org/0000-0002-7519-7894 \\ Universidade de Pernambuco, Brasil \\ E-mail: caiocgsilva@gmail.com \\ José Rodrigues Laureano Filho \\ ORCID: https://orcid.org/0000-0002-9645-2057 \\ Universidade de Pernambuco, Brasil \\ E-mail: laureano.filho@upe.br \\ Antônio Azoubel Antunes \\ ORCID: https://orcid.org/0000-0002-3500-3354 \\ Universidade de Pernambuco, Brasil \\ E-mail: antonio.antunes@upe.br \\ Gabriela Granja Porto Petraki \\ ORCID: https://orcid.org/0000-0002-4687-3697 \\ Universidade de Pernambuco, Brasil \\ E-mail: gabriela.porto@upe.br
}

\begin{abstract}
Resumo
Esse estudo objetiva-se avaliar os aspectos econômicos dos acidentes motociclísticos e o perfil epidemiológico dos acidentados antes e após a implementação da "Lei Seca". Para isso, foi realizada uma coleta de dados secundários sobre os acidentes com motocicletas no Brasil ocorridos entre 2003 e 2017 na plataforma DATASUS. Coletou-se informações do grupo Morbidade Hospitalar por Causas Eternas e das frotas anuais de motocicletas pelo Denatran. Os dados selecionados foram o local de internação, os valores absolutos anuais das autorizações de internações hospitalares pagas, valores médios por internações, valores totais gastos e médias de dias de internamento. As informações foram relacionadas com o sexo, faixa etária, as regiões do país e o ano de ocorrência. Os resultados mostraram que a ocorrência de acidentes foi maior nos homens, entre 20 e 29 anos, com mais de 300.000 internações no período entre 2003 e 2017. Os maiores valores estão concentrados na região Sudeste. A "Lei Seca" reduziu os números de autorizações de internações hospitalares no ano de sua implantação, porém, nos anos posteriores, os números aumentaram proporcionalmente com a frota de motocicletas. Entretanto, observou-se uma diminuição nos valores gastos, indicando possivelmente, a diminuição da gravidade dos acidentes. Assim, pode-se concluir que medidas de da fiscalização e punição dos infratores, bem como a educação e conscientização dos atuais e futuros condutores são importantes para reduzir efetivamente a quantidade de acidentes.
\end{abstract}

Palavras-chave: Motocicletas; Acidentes de trânsito; Hospitais; Economia.

\begin{abstract}
This study aims to evaluate the economic aspects of motorcycle accidents and the epidemiological profile of the injured before and after the implementation of the "Lei Seca". For this, a collection of secondary data on motorcycle accidents in Brazil occurring between 2003 and 2017 was conducted on the DATASUS platform. Information was collected from the group Hospital Morbidity by Eternal Causes and the annual motorcycle fleets by Denatran. The selected data were the place of hospitalization, annual absolute values of paid hospitalization authorizations, average values per hospitalization, total values spent, and average days of hospitalization. The information was related to gender, age group, the regions of the country, and the year of occurrence. The results showed that the occurrence of accidents was higher in men, between 20 and 29 years old, with more than 300,000 hospitalizations in the period
\end{abstract}


between 2003 and 2017. The highest figures are concentrated in the Southeast region. The "Lei Seca" reduced the numbers of authorizations for hospital admissions in the year of its implementation, but in subsequent years, the numbers increased proportionally with the motorcycle fleet. However, a decrease in the amounts spent was observed, possibly indicating a decrease in the severity of the accidents. Thus, it can be concluded that enforcement measures and punishment of offenders, as well as education and awareness of current and future drivers are important to effectively reduce the number of accidents.

Keywords: Motorcycles; Accidents, traffic; Hospitals; Economics.

\section{Resumen}

Este estudio pretende evaluar los aspectos económicos de los accidentes de moto y el perfil epidemiológico de los heridos antes y después de la implantación de la "Lei Seca". Para ello, se realizó una recopilación de datos secundarios sobre los accidentes de moto en Brasil ocurridos entre 2003 y 2017 en la plataforma DATASUS. La información se recogió del grupo Morbilidad Hospitalaria de Causas Eternas y de los parques de motocicletas anuales de Denatran. Datos seleccionados: el lugar de hospitalización, los valores absolutos anuales de las autorizaciones de hospitalización pagadas, los valores medios por hospitalización, los valores totales gastados y los días medios de hospitalización. La información estaba relacionada con el género, el grupo de edad, las regiones del país y el año de ocurrencia. Los resultados mostraron que la ocurrencia de accidentes fue mayor en los hombres, entre 20 y 29 años, con más de 300.000 hospitalizaciones en el período comprendido entre 2003 y 2017. Los valores más altos se concentran en la región del sureste. La "Lei Seca" redujo las cifras de autorizaciones de ingresos hospitalarios en el año de su implantación, pero en los años siguientes, las cifras aumentaron proporcionalmente con el parque de motos. Sin embargo, se observó una disminución de las cantidades gastadas, lo que posiblemente indica una disminución de la gravedad de los accidentes. Concluimos que medidas de aplicación de la ley y el castigo a los infractores, así como la educación y concienciación de los conductores son importantes para reducir efectivamente los accidentes.

Palabras clave: Motocicletas; Accidentes de trânsito; Hospitales; Economía.

\section{Introduçãa}

Anualmente, no mundo, os acidentes de transportes terrestres resultam em mais de 1.3 milhões de óbitos, além de deixar cerca de 20 a 50 milhões de pessoas com injúrias (OMS, 2018). As injúrias decorrentes desses eventos, em especial com os motociclistas, constituem um problema de saúde emergente, que vem provocando um forte impacto na população mundial, principalmente nos países emergentes (Pereira et al., 2013; Ul Baset et al., 2017).

Os acidentes de motocicletas são a etiologia mais comum para traumas de face, em especial as fraturas do complexo zigomático e mandíbula (Cavalcante et al., 2009; Porto et al., 2011; Albuquerque, 2012). Além disso, representam um grande perigo a saúde, que pode estar associado a morbidades significativas para os pacientes afetados e um custo socioeconômico para a sociedade (Lee et al., 2007).

As motocicletas devido ao seu formato, estrutura pequena e duas rodas, apresentam menor estabilidade e proteção aos seus condutores (Islam \& Brown, 2017). Como resultado, um condutor de uma motocicleta tem uma probabilidade 26 vezes maior de ter uma fatalidade do que o de um carro (NHTSA, 2015).

Os acidentes envolvendo motociclistas proporcionaram mais de 380.000 mortes anuais e $28 \%$ das fatalidades no mundo no ano de 2016 (Konlan et al., 2020). Segundo dados do DATASUS, no período de 2003 a 2013, houve um aumento de 271,2\% no número de mortes por essas circunstâncias de trânsito com motos no Brasil e, em 2012, foram registradas 80.831 vítimas com sequelas de desastres com motocicletas.

A associação da ingestão de álcool e direção é bastante frequente e perigosa, estando presente em muitas fatalidades no trânsito, sendo considerada um problema de saúde pública (Rehm et al., 2003). O álcool interfere nas respostas cognitivas e motoras, prejudicando a capacidade de resolver os problemas em situação de conflito. Diante da necessidade de reduzir a morbidade resultante dessa interação, foi promulgada a Lei $n^{\circ} 11.705$ de 19 de junho de 2008, popularmente conhecida como "Lei Seca", a qual é responsável pela proibição do consumo de bebida alcoólica por indivíduos que estejam conduzindo veículo automobilístico, podendo acarretar diversas penalidades (Detran, 2013). 
Dados que comprovem o impacto socioeconômico dos acidentes motociclísticos e sua relação com o consumo de álcool são escassos. Diante disso, o objetivo do presente trabalho foi realizar um levantamento epidemiológico das internações hospitalares decorrentes de acidente motociclísticos no Brasil cinco anos antes a implantação da "Lei Seca" e 9 anos após, que poderá servir de subsídio para o planejamento e implantação de políticas públicas voltadas para a prevenção e promoção da saúde.

\section{Metodologia}

O presente estudo observacional descritivo coletou os dados epidemiológicos dos acidentes motociclísticos no Brasil, ocorridos no período de 2003 a 2017. Lima-Costa e Barreto (2003), apontam que grandes bancos de dados secundários de amplitude nacional podem ser empregues para estudos observacionais descritivos. Os dados foram coletados de forma secundária no site do Ministério da Saúde- DATASUS. Foram obtidos os valores absolutos anuais das autorizações de internações hospitalares (AIH’s) pagas, valores médios por internações, valores totais gastos e médias de dias de internamento decorrentes de acidentes envolvendo motocicletas nos quais as vítimas terão que ser atendidas no Sistema único de Saúde (SUS) e seus dados foram computados pelo local de internação. Assim como foram observados a faixa etária e o sexo dos acidentados. Os resultados foram separados por região do estado e por período cinco anos antes do ano de 2008, período que ocorreu a implantação da "Lei Seca", e 9 anos após.

Além disso, foram catalogados dados da frota anual de motocicletas através do DENATRAN. Os dados foram coletados por meses e somados de acordo com seus anos e região no período de 2003 a 2017.

O presente trabalho está de acordo com a Resolução No 510/2016 CNS-CONEP, em seu artigo primeiro, se enquadrando nas pesquisas que utilizam informações de domínio público, não precisando, portanto, de avaliação do comitê de ética.

Os dados foram expressos através das medidas: média, desvio padrão (média \pm DP) e mediana, percentis 25 e 75 . Para avaliar diferença significativa entre os dois períodos, foi utilizado o teste t-Student ou Mann-Whitney.

A escolha do teste $t$-Student ocorreu nas situações em que foi verificada normalidade dos dados em cada período e o teste de Mann-Whitney quando foi rejeitada em pelo menos um dos períodos. A verificação da normalidade foi realizada pelo teste de Shapiro-Wilk e a igualdade de variâncias foi através do teste F de Levene.

Para avaliar a tendência e se existe diferença significativa entre os dois períodos (antes e após a implantação da lei seca) nas variáveis: número de AIH's, valores do gasto e relação (gasto/número de AIH's) os valores foram divididos pelo número médio da frota de motos por ano, ou equivalentemente se obteve as taxas das variáveis pelo número motos (média do número de motos por ano).

A margem de erro utilizada na decisão dos testes estatísticos foi de 5\%. Os dados foram digitados na planilha EXCEL e o programa utilizado para obtenção dos cálculos estatísticos foi o IMB SPSS na versão 23.

\section{Resultados}

A amostra foi composta por dados de 898.346 internamentos hospitalares por acidentes motociclísticos ao longo do periodo de 2003 a 2017. A maioria desses pacientes era do sexo masculino (84,987\%) e estava no grupo de 20 à 29 anos de idade $(40,603 \%)$.

A maior parte dos internamentos hospitalares por acidentes motociclísticos ocorreram na região Sudeste (43,099\%) seguida pela região Nordeste $(28,636 \%)$, enquanto a região Norte apresentou o menor número $(8,137 \%)$. Em todas as regiões geográficas do Brasil, a faixa etária mais prevalente foi dos 20 aos 29 anos de idade, com mais de 300.000 internações. 
Ao longo do periodo estudado, o número AIH's teve um valor crescente até o ano de 2008, ano de aplicação da Lei Seca, onde o número de AIH's estabilizou, e, após esse periodo, o número de AIH's teve uma curva ascendente como mostra o Gráfico 1.

Gráfico 1. Evolução do número de autorizações de internação hospitalares (AIHs) entre 2003 e 2017.

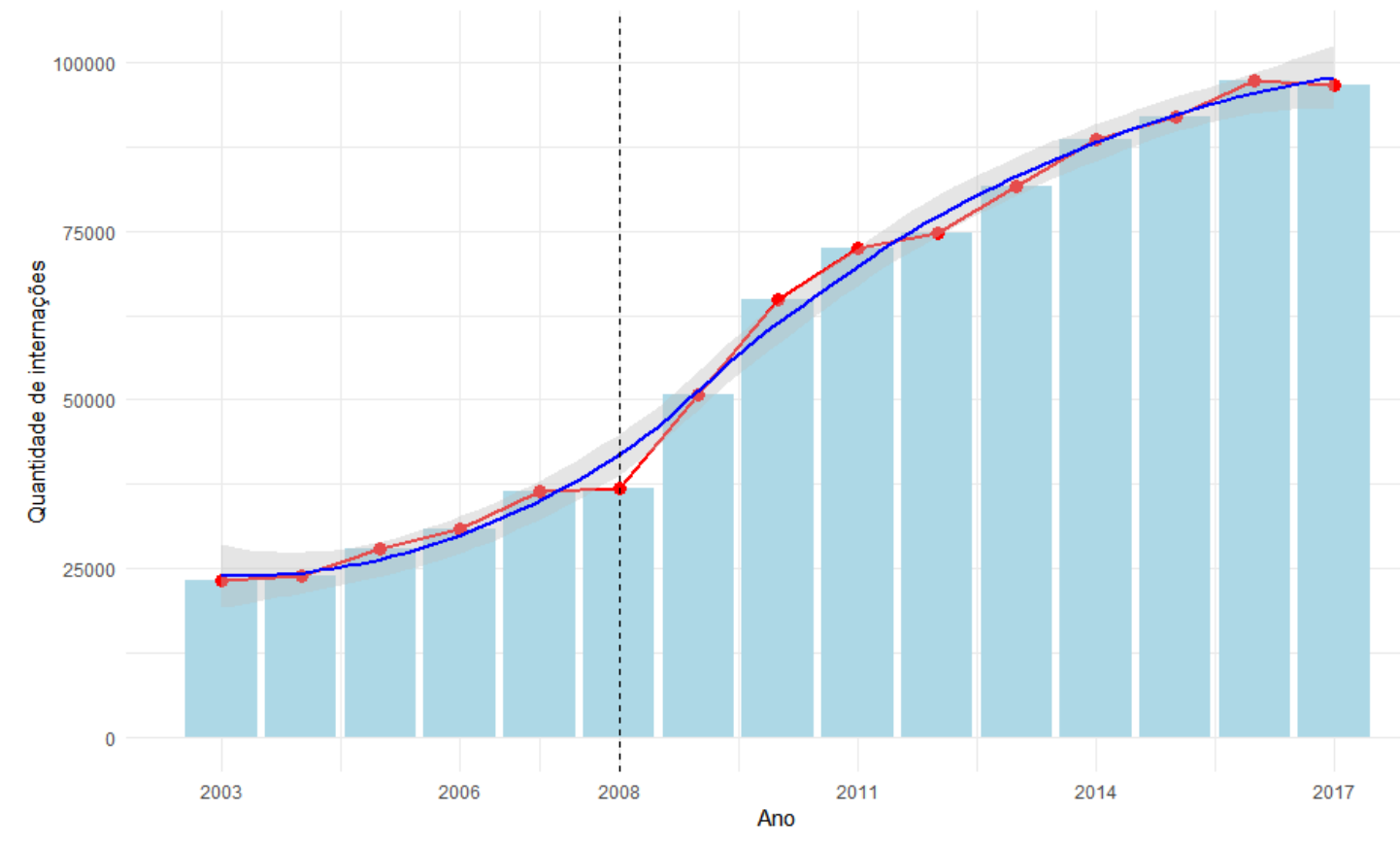

Fonte: Arquivo pessoal.

Na Tabela 1, podem ser observadas as estatísticas das variáveis AIH's, valor pago, relação gasto/número de AIH's e média de permanência no grupo total. Nesta tabela, verifica-se que a variabilidade foi elevada nas variáveis AIH's e valor pago, desde que os valores dos desvios padrão foram inferiores às médias correspondentes e foram reduzidos nas variáveis valor médio e média de permanência (desvios padrão inferiores a 1/3 das médias correspondentes).

Tabela 1. Estatística das variáveis: AIH's aprovadas, valor pago, valor médio gasto por paciente e média da permanência no grupo total.

\begin{tabular}{ccc}
\hline Variável & Média \pm DP & Mediana (P25; P75) \\
\hline AIH's aprovadas & $1197,79 \pm 1940,76$ & $197,00(13202,00 ; 494,50)$ \\
Valor pago $(\mathrm{R} \$)$ & $1500043,78 \pm 2680590,20$ & $189425,22(20768012,87 ; 559097,30)$ \\
Valor médio & $1096,55 \pm 334,00$ & $887,00(2171,41 ; 1063,27)$ \\
Média permanência & $6,27 \pm 0,94$ & $5,70(14,70 ; 6,20)$ \\
\hline
\end{tabular}

Fonte: Arquivo pessoal.

A Tabela 2 mostra que, exceto na variável "Dias de permanência", as médias e medianas das outras três variáveis foram correspondentemente mais elevadas no período após a Lei Seca do que antes da Lei Seca, enquanto, na variável média 
de permanência, a média foi mais elevada no período antes da Lei Seca. Foram registradas diferenças significativas entre os dois períodos.

Tabela 2. Estatísticas das variáveis: AIH aprovadas, valor pago, valor médio gasto por paciente e média da permanência hospitalizado por período antes da lei seca (2003 a 2007) e após a lei seca (2008 a 2017).

\begin{tabular}{|c|c|c|c|}
\hline Variável & $\begin{array}{c}\text { Antes da lei seca } \\
\text { 2003 a 2007 } \\
\text { Média } \pm \text { DP } \\
\text { Mediana (P25: P75) } \\
\end{array}$ & $\begin{array}{c}\text { Após a lei seca } \\
\text { 2008 a 2017 } \\
\text { Média } \pm \text { DP } \\
\text { Mediana (P25: P75) } \\
\end{array}$ & Valor de p \\
\hline AIH aprovadas & $\begin{array}{c}569,98 \pm 1103,20 \\
77,00(8925,00 ; 210,50)\end{array}$ & $\begin{array}{c}1511,70 \pm 2179,47 \\
321,25(13202,00 ; 713,50)\end{array}$ & $\mathrm{p}^{(1)}<0,001^{*}$ \\
\hline Valor pago $(\mathrm{R} \$)$ & $\begin{array}{c}571649,22 \pm 1190349,07 \\
62203,78(10308477,35 ; 192436,29)\end{array}$ & $\begin{array}{c}1964241,06 \pm 3070955,43 \\
332138,31(20768012,87 ; 807740,22)\end{array}$ & $\mathrm{p}^{(1)}<0,001^{*}$ \\
\hline Valor médio & $\begin{array}{c}895,07 \pm 289,22 \\
704,65(1796,41 ; 937,53)\end{array}$ & $\begin{array}{c}1197,30 \pm 308,55 \\
976,53(2171,41 ; 1156,76)\end{array}$ & $\mathrm{p}^{(1)}<0,001^{*}$ \\
\hline Média permanência & $\begin{array}{c}6,43 \pm 1,26 \\
5,70(14,70 ; 6,30)\end{array}$ & $\begin{array}{c}6,19 \pm 0,73 \\
5,70(9,20 ; 6,10)\end{array}$ & $\mathrm{p}^{(1)}=0,007^{*}$ \\
\hline
\end{tabular}

(*) Diferença significativa a 5\%. (1) Através do teste de Mann-Whitney. Fonte: Arquivo pessoal.

Os dados da Tabela 3 mostram que as médias das variáveis AIH's por 1000 motos variaram de 3.69 a 3.84 e as dos valores pagos por moto variaram de $\mathrm{R} \$ 3.66$ a $\mathrm{R} \$ 4.94$, sendo correspondentemente mais elevadas no período após a lei seca, enquanto a média da relação gastos/número de AIH's foi mais elevada antes da lei seca $(5,90$ x 3,19). Para a margem de erro fixada foram verificadas diferenças significativas $(\mathrm{p}<0,05)$ entre os dois períodos nas variáveis: valor pago e na relação (gasto por AIH's) por 1000 motos.

Tabela 3. Estatísticas das taxas: número total de AIHs aprovadas por 1000 motos, total valor pago por moto e total da relação (gasto por AIH's) por 1000 motos por período.

\begin{tabular}{|c|c|c|c|}
\hline Variável & $\begin{array}{c}\text { Antes da lei seca } \\
2003 \text { a } 2007 \\
\text { Média } \pm \text { DP } \\
\text { Mediana (P25: P75) } \\
\end{array}$ & $\begin{array}{c}\text { Após a lei seca } \\
\text { 2008 a 2017 } \\
\text { Média } \pm \text { DP } \\
\text { Mediana (P25: P75) } \\
\end{array}$ & Valor de $p$ \\
\hline $\begin{array}{l}\text { AIH's aprovadas/ } \\
1000 \text { motos }\end{array}$ & $\begin{array}{c}3,69 \pm 0,22 \\
3,63(3,54 ; 3,88)\end{array}$ & $\begin{array}{c}3,84 \pm 0,33 \\
3,95(3,77 ; 4,01)\end{array}$ & $\mathrm{p}^{(1)}=0,172$ \\
\hline Valor pago por moto & $\begin{array}{c}3,66 \pm 0,15 \\
3,60(3,53 ; 3,82)\end{array}$ & $\begin{array}{c}4,94 \pm 0,58 \\
5,20(4,80 ; 5,27)\end{array}$ & $\mathrm{p}^{(1)}=0,012 *$ \\
\hline $\begin{array}{l}\text { Relação (gasto/ } \\
\text { Número de AIHs) }\end{array}$ & $\begin{array}{c}5,90 \pm 0,87 \\
6,17(5,00 ; 6,68)\end{array}$ & $\begin{array}{c}3,19 \pm 0,57 \\
2,94(2,71 ; 3,68)\end{array}$ & $\mathrm{p}^{(2)}<0,001 *$ \\
\hline
\end{tabular}

(*) Diferença significativa a 5\%. (1) Através do teste de Mann-Whitney. (2) Através do teste t-Student com variâncias iguais. Fonte: Arquivo pessoal.

Nos gráficos 2 e 3 pode-se observer que, após o ano de 2008, os números apresentaram níveis próximos, demonstrando um aumento proporcional entre a frota de motocicletas e internações. 
Gráfico 2. Taxa do total de AIHS por 1000 motos no grupo total por ano.

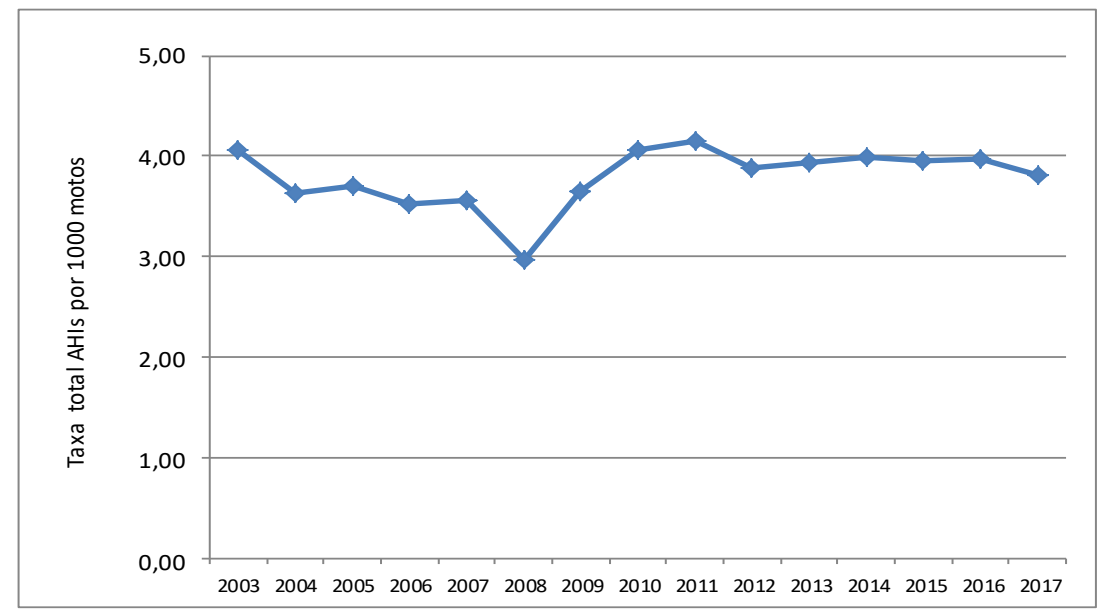

Fonte: Arquivo pessoal.

Gráfico 3. Taxa do total gasto por cada moto no grupo total por ano.

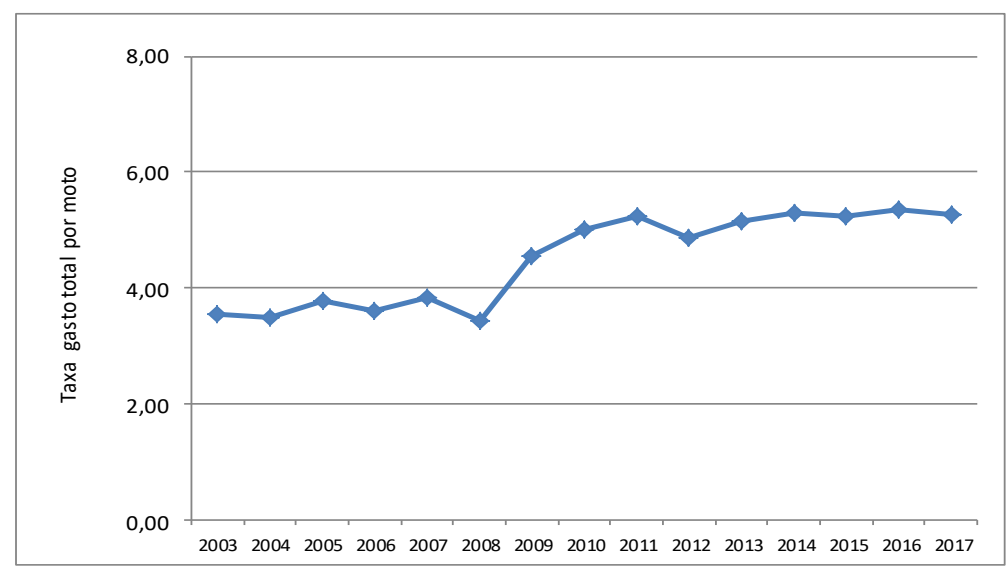

Fonte: Arquivo pessoal.

\section{Discussão}

Com o crescimento da frota de motocicletas, os ocupantes desses veículos vêm, paulatinamente, assumindo o primeiro lugar entre as vítimas de acidentes de trânsito com veículos a motor (Brasileiro, Vieira \& Silveira, 2010). Dentre os fatores para os elevados índices de acidentes motociclísticos estão o aumento do número de motocicletas, o trânsito frenético e principalmente a imprudência por parte dos condutores, provocando uma sobrecarga para o sistema de saúde e, por consequência, para toda sociedade. O crescimento do uso das motocicletas é justificado pelos seus benefícios em relação aos automóveis, como agilidade, economia e melhor desenvoltura no trânsito; o que torna a motocicleta um meio de transporte socialmente importante (Salvaro, 2012).

Em nosso estudo, com relação às internações hospitalares decorrentes de acidentes motociclísticos, houve uma predominância do sexo masculino, sendo um dado consensual na maioria da literatura referenciada (Santos et al., 2008; Brasileiro et al., 2010; Derry et al., 2017; Islam \& Brown, 2017; Baru et al., 2019; Porto et al., 2020; Cavalcante et al.; 2021). Isso pode ser explicado pelo fato de o homem representar o maior percentual de indivíduos usuários de motocicletas como meio de transporte, estando, assim, mais sujeitos a acidentes.

A distribuição da faixa etária mostrou que a maioria da população internada em ambiente hospitalar por acidente motociclístico foi de adultos jovens com idade entre 20 e 29 anos, assim como nos estudos de Baru et al. 2019, Brasileiro, Vieira e Silveira (2010) e Lee et al. (2007) que mostraram que a população incluída nessa faixa etária foi mais acometida por 
esses incidentes. Fato que pode ser explicado pela maior atividade dessa faixa etária, expondo-se mais a fatores de risco e com um perfil menos prudente quanto aos hábitos de dirigir, muitas vezes inconsequente, pela própria idade.

Derry et al. (2017) em um estudo transversal observaram o risco de mortalidade em acidentes de trânsito no norte de Gana. Os dados foram coletados de relatórios policiais entre os anos de 2007 e 2011. Os acidentes de trânsito foram considerados extremamente graves, já que 35\% de todas as colisões foram fatais. O índice de mortalidade no sexo masculino foi seis vezes mais elevado do que o do feminino. Sendo sugerida pelo estudo a aplicação das leis de trânsito, particularmente em relação ao uso de capacete, excesso de velocidade e uso de álcool. Bem como, uma educação em segurança do trânsito para criar condutores mais responsáveis.

Desse modo, esses dados nos levam a refletir sobre os padrões socioculturais, em relação a questões de sexo, que se perpetuam em nossa sociedade e terminam por conduzir a altas taxas de morbimortalidade em adultos jovens do sexo masculino, no auge de suas capacidades, acarretando prejuízos econômicos consideráveis à nação (Santos et al., 2008).

As motocicletas estão associadas as novas modalidades de comércio em regiões urbanas e rurais (Konlan et al., 2020). Seu custo baixo, economia de combustível e manutenção e a versatilidade no trânsito, proporciona um aumento do número de motoboys e mototaxistas (Brasileiro et al., 2010). Nas áreas de maior densidade demográfica, com alto impacto comercial e trânsito intenso, existem mais profissionais que utilizam esse veículo. as motocicletas apresentam uma menor estabilidade, são menos visíveis no trânsito e não tem uma proteção física como o carro. Tornando os usuários deste transporte mais vulneráveis a lesões durante uma colisão (Organização Pan-Americana de Saúde - OPAS/OMS, 2007). Assim, a frequência de acidentes e hospitalizações podem aumentar, como é o caso de maior ocorrência nas regiões Sudeste e Nordeste do Brasil.

O uso de álcool está associado à adoção, por parte do condutor, de fatores de risco importantes nos acidentes motociclísticos, incluindo excesso de velocidade, falta de coordenação motora e ausência do capacete. Em 2008, um estudo realizado por Santos et al. (2008) no Piauí, referente ao perfil das vítimas de trauma por acidente de moto observou que entre as vítimas de acidente, condutores de moto com antecedente de ingestão de álcool, 71,2\% não utilizavam capacete no momento do acidente, enquanto entre aqueles sem antecedente de ingestão, esta proporção foi de 43,3\%. Assim, observou-se uma chance quatro vezes maior de não utilização desse equipamento de segurança entre aqueles que usavam bebida alcoólica. Islam e Brown (2017) realizaram uma análise, através de dados policiais, da gravidade das lesões de acidentes com motocicletas no estado norte-americano do Alabama. No estudo foram observados que fatores como influência de álcool, ausência de capacete e alta velocidade eram estatisticamente significativos nos acidentes com motocicletas.

Porto et al. (2020) em um estudo prospectivo analisaram a influência de variáveis como sexo, idade, uso e tipo de capacete, acidentes prévios, consumo de álcool com relação a traumas em face e tempo de internação decorrentes de acidentes motociclísticos. A amostra foi composta por 455 pacientes sendo a maioria do sexo masculino (90,8\%) e entre os 18 e 29 anos (54,5\%). Dos pacientes, 36,5\% relataram não usar capacete e 31,6\% relataram usar capacete aberto. O uso de álcool foi relatado em 38,7\% do grupo. Em 79,8\% da amostra, o uso de álcool foi classificado como de baixo risco. Esses pacientes também tenderam a permanecer hospitalizados por mais de 10 dias. A utilização do capacete pelo motorista ou acompanhante está associada com a severidade de uma injúria resultante do acidente e, para aquele que não o usam, existe uma chance cinco vezes maior de se acidentar gravemente, se compararmos com quem o coloca (Baru et al., 2019; Cavalcante et al., 2021).

No presente estudo, pode-se observar que o número de AIH's apresentou uma curva ascendente no período de 2003 a 2017, como mostra o Gráfico 1 e isto se deve ao aumento proporcional da frota de motocicletas. Lee et al. (2007) demonstrou que existe uma associação estatisticamente significativa entre acidentes de veículos e a necessidade de intervenção ativa. Além disso, no nosso estudo, foi possível constatar que as internações aumentam na mesma proporção que a frota de motociclistas, o que pode indicar uma falha na fiscalização e na educação dos motociclistas mais recentes, bem como dos antigos. 
Os custos da hospitalização podem envolver a internação, intervenções cirúrgicas, medicação entre outros, pagos pela economia pública (Miki et al., 2014). Porto et al. (2021) concluiu com sua pesquisa que vítimas de acidentes motociclísticos cujo equipamento de proteção não foram utilizados e com ingestão alcoolica tiveram custos hospitalares maiores e com mais gravidade.

Observa-se com os resultados apresentados nesta pesquisa, que a média de tempo de internamento foi de, aproximadamente, 6 dias durante todo o periodo (2003 a 2017), tendo uma diminuição do valor pago e valor médio gasto por paciente no período pré “lei seca. A relação gasto/número de AIH’s diminuíram no periodo após "Lei Seca” (2008 a 2017). Isso sugere que os quadros clínicos dos indivíduos foram menos graves em relação ao período antes da implementação da "Lei Seca".

Fatores climáticos, condições da estrada, aspectos socioculturais e econômicos variam de acordo com os países e, assim, pode existir uma limitação desse estudo para outras populações. Entretanto, os resultados encontrados podem servir de base e norteamento para a realização de pesquisas e como estímulo para criação de medidas preventivas relacionadas aos acidentes de trânsito em todo o mundo.

\section{Considerações Finais}

Podemos concluir que existem altos índices de internações hospitalares, principalmente entre homens de 20 a 29 anos, residentes nas regiões Sudeste e Nordeste do Brasil e que medidas de medidas de fiscalização e punição dos infratores, bem como a educação e conscientização dos atuais e futuros condutores são importantes para reduzir efetivamente a quantidade de acidentes nesse público e em toda a sociedade.

Para estudos futuros, recomendamos os observacionais prospectivos, que verifiquem a ingestão de bebida alcoolica com a realização de testes bioquímicos quando o motociclista der entrada na emergência.

\section{Agradecimentos}

Este estudo foi parcialmente apoiado pela Fundação de Amparo a Ciência e Tecnologia de Pernambuco (FACEPE).

\section{Referências}

Albuquerque, T. T. P. (2012). Fixação de fratura do osso zigomático em único ponto: sutura frontozigomática. 42 f. Monografia (Especialização em Cirurgia e Traumatologia Buco-Maxilo-Facial) - Universidade Estadual da Paraíba.

Baru, A., Azazh, A., \& Beza, L. (2019). Injury severity levels and associated factors among road traffic collision victims referred to emergency departments of selected public hospitals in Addis Ababa, Ethiopia: the study based on the Haddon matrix. BMC emergency medicine, 19(1), 1-10. https://doi.org/10.1186/s12873-018-0206-1

Brasileiro, B. F., Vieira, J. M., \& Silveira, C. E. S. D. (2010). Avaliação de traumatismos faciais por acidentes motociclísticos em Aracaju/SE. Rev. Cir. Traumatol. Buco-Maxilo-fac. 10 (2), 97-104.

Cavalcante, D. K. F., Veloso, S. R. M., de Almeida Durão, M., de Carvalho Melo, V., de Melo Monteiro, G. Q., \& Porto, G. G. (2021). Do Helmet Use and Type Influence Facial Trauma Occurrence and Severity in Motorcyclists? A Systematic Review and Metanalysis. Journal of Oral and Maxillofacial Surgery. 79 (7), 1492-1506. https://doi.org/10.1016/j.joms.2021.02.028

Cavalcante, J. R., Guimarães, K. B., Vasconcelos, B. C. D. E., \& Vasconcellos, R. J. D. H. (2009). Estudo epidemiológico dos pacientes atendidos com trauma de face no Hospital Antônio Targino-Campina Grande/Paraíba. Brazilian Journal of Otorhinolaryngology, 75, 628-633. https://doi.org/10.1590/S1808-86942009000500003

DATASUS. (2016). Morbidade Hospitalar do SUS por Causas Externas - por local de internação. Datasus. http://tabnet.datasus.gov.br/cgi/deftohtm.exe?sih/cnv/nrpb.def.

DETRAN-PE. Frota registrada segundo o tipo - Pernambuco. http://www.detran.pe.gov.br/images/stories/estatisticas/HP/1.7_frota_pe.pdf.

Damsere-Derry, J., Palk, G., \& King, M. (2017). Road accident fatality risks for "vulnerable" versus "protected" road users in northern Ghana. Traffic injury prevention, 18(7), 736-743. https://doi.org/10.1080/15389588.2017.1302083 
Research, Society and Development, v. 11, n. 2, e20611225614, 2022

(CC BY 4.0) | ISSN 2525-3409 | DOI: http://dx.doi.org/10.33448/rsd-v11i2.25614

Islam, S., \& Brown, J. (2017). A comparative injury severity analysis of motorcycle at-fault crashes on rural and urban roadways in Alabama. Accident Analysis \& Prevention, 108, 163-171. https://doi.org/10.1016/j.aap.2017.08.016

Konlan, K. D., Doat, A. R., Mohammed, I., Amoah, R. M., Saah, J. A., Konlan, K. D., \& Abdulai, J. A. (2020). Prevalence and pattern of road traffic accidents among commercial motorcyclists in the Central Tongu District, Ghana. The Scientific World Journal, 2020. https://doi.org/10.1155/2020/9493718

Lee, K. H., Snape, L., Steenberg, L. J., \& Worthington, J. (2007). Comparison between interpersonal violence and motor vehicle accidents in the aetiology of maxillofacial fractures. ANZ journal of surgery, 77(8), 695-698. https://doi.org/10.1111/j.1445-2197.2007.04189.x

Lima-Costa, M. F., \& Barreto, S. M. (2003). Tipos de estudos epidemiológicos: conceitos básicos e aplicações na área do envelhecimento. Epidemiologia e serviços de saúde, 12(4), 189-201. https://doi.org/10.5123/S1679-49742003000400003

Miki N, Cabrera Martimbianco AL, Tomomi Hira L, Landim Lahoz G, Alvachian Fernandes HJ, Baldy dos Reis F. (2014) Profile of trauma victims of motorcycle accidents treated at hospital São Paulo. Acta Ortop Bras. 22: 219-22. https://doi.org/10.1590/1413-78522014220400642

NHTSA. (2015). Traffic Safety Facts - 2015 Data. National Highway Traffic Safety Administration. https://www.nhtsa.gov/document/traffic-safety-facts2015-data

Organização Pan-Americana da Saúde - OPAS/OMS. (2007) Helmets: a road safety manual for decision-makers and practitioners. http://whqlibdoc.who.int/publications/2006/9241562994_por.pdf

Pereira, R. C. F., Galvão, P. V. M., Nogueira, C. D. M., Vasconcelos, V. S., Campelo, R. I. C., \& Souza, E. H. A. D. (2013). Acidentes fatais com motociclistas no Recife e suas repercussões faciais. Revista de Cirurgia e Traumatologia Buco-maxilo-facial, 13(4), 101-108.

Porto DE, Araújo JMDN, Júnior CL, Andrade ESS. (2021) Pattern of Maxillofacial Trauma and Associated Factors in Traffic Accident Victims. J Craniofac Surg. 32(3):1010-1013. https://doi.org/10.1097/SCS.0000000000007002

Porto, D. E., Tavares, G. R., Tavres, S. S. S., \& Cavalcante, J. R. (2011). Perfil epidemiológico dos traumatismos faciais de pacientes atendidos em hospitais de emergência e trauma de Campina Grande-PB. IJD. International Journal of Dentistry, 10(4), 209-222.

Porto, G. G., de Menezes, L. P., Cavalcante, D. K. F., de Souza, R. R. L., Carneiro, S. C. D. A. S., \& Antunes, A. A. (2020). Do type of helmet and alcohol use increase facial trauma severity? Journal of Oral and Maxillofacial Surgery, 78(5), 797-e1. https://doi.org/10.1016/j.joms.2019.12.004

Rehm, J., Gmel, G., Sempos, C. T., \& Trevisan, M. (2003). Alcohol-related morbidity and mortality. Alcohol Research \& Health, 27(1), 39.

Salvaro, João Carlos. (2012) Direção defensiva para motociclistas.

Santos, A. M. R. D., Moura, M. E. B., Nunes, B. M. V. T., Leal, C. F. D. S., \& Teles, J. B. M. (2008). Profile of motorcycle accident victims treated at a public hospital emergency department. Cadernos de saude publica, 24(8), 1927-1938. https://doi.org/10.1590/S0102-311X2008000800021

Ul Baset, M., Rahman, A., Alonge, O., Agrawal, P., Wadhwaniya, S., \& Rahman, F. (2017). Pattern of road traffic injuries in rural Bangladesh: burden estimates and risk factors. International journal of environmental research and public health, 14(11), 1354. https://doi.org/10.3390/ijerph14111354

World Health Organization. (2018). Global status report on road safety 2018: summary (No. WHO/NMH/NVI/18.20). World Health Organization. 